\title{
Endothelial Dysfunction in Breast Cancer In-Vivo Model ${ }^{+}$
}

\author{
Buğra Elkar ${ }^{1}$, Mustafa Barış ${ }^{2}$, Meryem Çalışır ${ }^{3}$, Yasemin Çakır ${ }^{4}$, Safiye Aktaş ${ }^{4}$, Merve Tütüncü ${ }^{4}$, \\ M. Kürşat Şimşek ${ }^{2}$, Mustafa Seçil ${ }^{2}$, Osman Y1lmaz ${ }^{3}$ and Zekiye Altun ${ }^{4, *}$ \\ 1 School of Medicine, Dokuz Eylul University, Izmir 35340, Turkey; bgrelkar@gmail.com \\ 2 Department of Radiology, School of Medicine, Dokuz Eylul University, Izmir 35340, Turkey; \\ mustafambaris@yahoo.com (M.B.); mkursatsimsek9@gmail.com (M.K.Ş.); mustafa.secil@deu.edu.tr (M.S.) \\ 3 Department of Laboratory Animals, Institute of Health Sciences, Dokuz Eylul University, Izmir 35340, \\ Turkey; meryemcalisir@gmail.com (M.Ç.); osman.yilmaz@deu.edu.tr (O.Y.) \\ 4 Department of Basic Oncology, Institute of Oncology, Dokuz Eylul University, Izmir 35340, Turkey; \\ yasemin.cakir@yahoo.com (Y.Ç.); safiyeaktas@gmail.com (S.A.); mervetutuncu@gmail.com (M.T.) \\ * Correspondence: zekiye.altun@deu.edu.tr; Tel.: +90-232-4128204 \\ + Presented at the 2nd International Cell Death Research Congress, Izmir, Turkey, 1-4 November 2018. \\ Published: 6 December 2018
}

\begin{abstract}
Although the endothelial dysfunction is related with tumor development, there is no consensus on the suppressive or supportive effect on tumor growth. The goal of the present study was to evaluate endothelial dysfunction related factors in animal breast cancer model that was developed by administrating endothelial nitric oxide synthase blocking agent, Nitro-L-arginine methyl ester hydrochloride (L-NAME). Endothelial dysfunction related main factors such as nitric oxide synthase, interleukin-6, vascular endothelial growth factor receptor-2 and vascular endothelial cadherin were investigated by immunohistochemically in tumor and carotid artery tissues. In tumor tissues apoptosis was determined by TUNEL assay. The level of endothelin-1 in blood was measured by ELISA. İntima-media thickness of carotid artery was evaluated with Doppler-USG measurements. As a result, in this study it was shown that vascular endothelial growth factor receptor-2, endothelin-1, endothelial nitric oxide synthase, interleukin-6, vascular endothelial cadherin and E-selectin molecules play a role in reducing breast tumor growth based on endothelial dysfunction.
\end{abstract}

Keywords: endothelial dysfunction; breast cancer; USG; L-NAME; cancer development

\section{Introduction}

Endothelial dysfunction (ED) is an effective factor in tumor development. The relationship between the tumor cell and endothelium is constructed by complex biological signals of tumor microenvironment which is generated by the endothelial cells, tumor-related fibroblasts and extracellular matrix. In this environment, interleukin-6 (IL-6), E-selectin, vascular endothelial cadherin (VE-cadherin), NF- $\mathrm{B}$ and target genes may be altered in response to possible tumor growth and inflammation [1]. In addition, increased intima-media thickness (IMT) screening by Doppler ultrasonography (US) has been accepted as a sign of ED in experimental models [2].

The aim of this study was to determine whether ED induced by different doses of nitrous oxide synthase (NOS) inhibition would have a possible preventive effect on the development of metastatic breast cancer (BC). For this purpose, NOS inhibition was performed in nude mice by increasing doses of intraperitoneal L-NAME injection and then BC cells were injected for development of xenograft breast cancer. The effects of ED on the possible tumor development were related to inflammation 
investigated with Endothelin, IL-6, E-selectin, VE-cadherin and NF-kB expressions in tumor tissues. Endothelin-1 (ET-1) and e-NOS expressions was also determined in carotid artery tissues.

\section{Materials and Methods}

Female nude-mice (5-7 weeks, $25 \mathrm{~g}$ ) were used (seven rats/groups). At the beginning, the animals were divided into following groups: (0) Control group injected with serum Physiological (SF), (1) injected with $185.92 \mu \mathrm{Mol} / \mathrm{kg}$ L-NAME, (2) injected with $92.5 \mu \mathrm{Mol} / \mathrm{kg}$ L-NAME, (3) injected with $61.66 \mu \mathrm{Mol} / \mathrm{kg}$ L-NAME. All protocols were approved by the ethics committee for the care and use of laboratory animals.

Animals were administered intraperitoneal SF (in control group) and L-NAME in other groups twice a day, for 7 days. IMT was evaluated before and after 8th day of L-NAME injection by USG. On the 8th day of the study, in order to form breast tumor, equal numbers $(1 \times 106 / \mathrm{mL})$ of $4 \mathrm{~T} 1 \mathrm{BC}$ cells were injected into subcutaneous region. The tumor size was measured with caliper every day. On the 21st day of the study, mice were sacrificed, tumor tissues and carotid arteries were removed for morphological evaluation and immunohistochemical staining and also plasma samples were obtained for biochemical studies. In plasma samples endothelin-1 were studied using ELISA. In tumor tissues apoptosis was studied by TUNEL method. Furthermore, ET-1, vascular endothelial growth factor-2 (VEGFR2), eNOS, VE-Cadherin, NF- $\kappa$ B, endothelial-selectin (e-Selectin)/CD62E and IL-6 was studied immunohistochemically. Staining were scored as no staining (0), 0-33\% staining (+), $33-66 \%$ staining (++), $66-100 \%$ staining (+++) in tumor tissues. In carotid arteries staining's were scored no staining (0), 0-50\% staining (+), 50-100\% staining (++) for Endothelin and no staining (0), focal staining $(+)$, diffuse weak staining (++), diffuse moderate staining (+++) and diffuse strong staining (++++) for e-NOS. The results were evaluated statistically using Mann-Whitney-U test in SPSS 22.0 program at $p<0.05$ level.

\section{Results}

According to groups, mean tumor volumes were as follows: (0) $676.43 \pm 164.94$, (1) $743.14 \pm$ 304.25 , (2) $844.43 \pm 439.39$, (3) $15.67 \pm 8.89 \mathrm{~mm} 3$. Compared to control group and other groups, there was a statistically significant decrease were determined at tumor volume in group 3 ( $p=0.0001$ ). Mean values of IMT increase were $0.0033 \pm 0.0026,0.0024 \pm 0.011,0.0120 \pm 0.009$ and $0.0110 \pm 0.003$ $\mathrm{mm}$ in groups respectively $(0,1,2,3)$. There was a statistically significant increase in group 3 when compared to the control group $(p=0.002)$. Two different measurements were carried out as intima/media thickness ratio and intima/full thickness ratio. Intima/media thickness and Intima/full thickness ratios were found as (0) 0.57 , (1) 0.60 , (2) 0.60 , (3) 0.71 and (0) 0.17 , (1) 0.18 , (2) 0.17 , (3) 0.23 respectively. Compared to the control group, there was a statistically significant increase determined at the results of both measurements in group $3(p=0.027$ and $p=0.020)$. Apoptosis percentages of the tumors were as follows: (0) 14.43, (1) 12.29, (2) 14.14, (3) 4.17 and there was a statistically significant decrease in Group 3 compared to the control group $(p=0.005)$. Microscopically measured necrosis percentages were found as (0) $25.14,(1) 26.14,(2) 11.71$, (3) 1.67 and there was a statistically significant decrease in Group 3 compared to the control group $(p=0.011)$. Liver, kidney, brain, lung and organ vessels were found to have normal histopathological findings $(p>0.05)$. Plasma endothelin-1 levels were as follows: (0) 27.44, (1) 27.13, (2) 29.68, (3) $28.11 \mathrm{pg} / \mathrm{mL}$ and no difference between groups ( $p>0.05$ ).

No significant difference was found between the groups in Endothelin-1 expression in IHC examinations of carotid arteries $(p>0.05)$. e-NOS expression was statistically lower in Group 3 compared to the control group of carotid arteries $(p=0.006)$.

IHC results of tumors: Endothelin-1 expression was statistically increased in all groups compared to the control group ( $p=0.000 ; p=0.000 ; p=0.000$ groups, respectively). VEGFR2 expression was statistically higher in all groups compared to the control group $(p=0.000 ; p=0.006 ; p=0.001$, respectively). e-NOS expression increased statistically in all groups compared to the control group ( $p$ $=0.000 ; p=0.023 ; p=0.000$ group, respectively).

VE-cadherin expression was statistically higher in Group 1 and Group 3 than in the control group $(p=0.004 ; p=0.218 ; p=0.000$ groups, respectively). Although no statistically significant 
difference was found in NF-k $\beta$ expression between groups, there was a decrease in each group ( $p=$ $0.454 ; p=0.139 ; p=0.503$, respectively). E-selectin/CD62E expression was statistically increased in Group 3 compared to the control group ( $p=0.025$ group, respectively). IL-6 expression was statistically higher in Group 3 compared to the control group $(p=0.000)$.

\section{Discussion and Conclusions}

ED have been associated with tumor growth, progression and metastasis [1]. In this study, ED was developed in BC model and the presence of ED has been proven by the presence of increased IMTs and decreased e-NOS expression in the carotid arteries. Furthermore, in ED group (group 3), approximately $98 \%$ reduction in tumor size confirmed our hypothesis by preventing tumor development.

In the group given the highest dose L-NAME tumor size was increased, while in the group given the lowest dose was $98 \%$ decrease. Tumor dimensions and apoptotic cell death was decreased in the ED group. It is suggested that the decrease in tumor growth is due to the increased expression of endotelin-1, VEGFR-2, e-NOS, IL-6, VE-cadherin and E-selectin in tumor tissues.

It was shown that there is a difference of IL-6 and E-selectin expressions between L-NAME applied groups and these molecules were thought to be responsible for the decrease in tumor growth in Group 3.

Molecules that we work with in tumor tissues were affected in all groups suggested that endothelial dysfunction should be small to be effective. Because, in tumor tissues where endothelial damage is greatly increased, tumor cells and microenvironment are thought to be stimulated to support tumor development. These questions should be clarified with more studies that are detailed. L-NAME-induced BC induced ED showed a decrease in tumor growth. The results of this study show that VEGFR-2, endothelin-1, e-NOS, IL-6, VE-cadherin and E-selectin molecules are involved in the reduction of tumor growth based on ED. In addition, because the nude mice used do not have cellular immunity, studies in animals with cellular immunity will probably further reduce or even prevent tumor growth. With the enlightenment of ED-related mechanisms, it will be possible for prevention from $\mathrm{BC}$ formation and to plan the development of new anticancer agents for $\mathrm{BC}$ treatment.

These results are potentially important for the development of protective vaccines for $\mathrm{BC}$. We anticipate that these results will be obtained in other cancers.

Acknowledgments: This study was financially supported Dokuz Eylul University Project-DEUKBSAG031.

\section{References}

1. Franses, J.W.; Drosu, N.C.; Gibson, W.J.; Chitalia, V.C.; Edelman, E.R. Dysfunctional endothelial cells directly stimulate cancer inflammation and metastasis. Int. J. Cancer 2013, 133, 1334-1344, doi:10.1002/ijc.28146.

2. Halcox, J.P.; Donald, A.E.; Ellins, E.; Witte, D.R.; Shipley, M.J.; Brunner, E.J.; Marmot, M.G.; Deanfield, J.E. Endothelial function predicts progression of carotid intima-media thickness. Circulation 2009, 119, 10051012, doi:10.1161/CIRCULATIONAHA.108.765701.

(C) 2018 by the authors. Licensee MDPI, Basel, Switzerland. This article is an open access article distributed under the terms and conditions of the Creative Commons Attribution (CC BY) license (http://creativecommons.org/licenses/by/4.0/). 